\title{
Obstructive Sleep Apnea and Sudden Cardiac Death: Is the Landscape Clear?
}

\author{
Emmanuel N. Simantirakis Panteleimon E. Papakonstantinou \\ Department of Cardiology, University Hospital of Heraklion, School of Medicine, University of Crete, \\ Heraklion, Crete, Greece
}

Obstructive sleep apnea (OSA) is associated with an increased risk of arrhythmogenesis, a fact that was recently recognized by the European Society of Cardiology (ESC) Guidelines concerning the management of patients with ventricular arrhythmias (VA) and prevention of sudden cardiac death (SCD) [1]. The most common arrhythmias observed in patients with OSA are sinus pause, sinus bradycardia, an increased rate of premature ventricular contractions, first-degree and Mobitz I second-degree atrioventricular block [1]. OSA has also been associated with an increased incidence of atrial fibrillation [2]. It is important to note that the frequency of arrhythmias, mainly nocturnal, increases with increased severity of OSA [2]. ESC recommends that OSA should be considered in the differential diagnosis of bradyarrhythmias (IIa,B) $[1,3]$. A circadian pattern of life-threatening VA and a high rate of SCD during sleeping hours (midnight to $6 \mathrm{am}$ ) were recorded [4-6] in OSA patients. It was also observed that patients with an implantable cardiac defibrillator and OSA had an increase in the onset of VA during sleep time [6].

\section{KARGER}

(๑) 2018 S. Karger AG, Basel

E-Mail karger@karger.com

www.karger.com/crd
However, this association is not clearly understood and several pathophysiological mechanisms have been proposed to explain how OSA leads to arrhythmogenesis and SCD [7]. An intriguing question that has arisen is whether OSA may lead to SCD in the absence of established cardiovascular disease.

The prevalence of OSA, defined as an apnea-hypopnea index $\geq 5$, was a mean of $17 \%$ (range $4-50 \%$ ) in women and $22 \%$ (range 9-37\%) in men in 11 large studies published between 1993 and 2013 [8]. In fact, the prevalence of arrhythmias depends on the comorbidities present in different populations [1]. OSA is highly prevalent in patients with cardiovascular diseases and is associated with a profile of perturbations that include oxidative stress, intermittent hypoxia, endothelial dysfunction and sympathetic activation [9]. Although OSA is associated with increased morality, its association with SCD requires further research [1]. Busselton Health Study [10] demonstrated that moderate-to-severe sleep apnea is independently associated with an increased risk of all-cause mor- 
tality in the studied population, while the Wisconsin Sleep Cohort [11] showed that all-cause mortality risk was significantly increased with OSA severity, independent of patients' age, sex and BMI. Gami et al. [4], in a large population of 10,701 adults, observed that the nocturnal hypoxemia, which is an important pathophysiological feature of OSA, strongly predicted SCD independent of well-established risk factors. Based on research findings, the authors proposed OSA as a novel risk factor for SCD. The above data are important, especially if we consider the great prevalence of OSA in middle-aged adults (30-60 years old), which was $24 \%$ for men and $9 \%$ for women [12].

ESC recommends that the presence of OSA and reduced oxygen saturation may be considered as a risk factor for SCD in subjects with sleep-disordered breathing $(\mathrm{IIb}, \mathrm{C})[1,4]$. Nevertheless, the strength of this recommendation is relatively low, and the data supporting it are weak, based mainly on small studies, retrospective studies and consensus meetings of experts. The significance of continuous positive airway pressure treatment (CPAP) in OSA patients for the prevention of VA is not well established, and it is uncertain if the appropriate treatment of OSA prevents the need for an implantation of a permanent pacemaker in patients with OSA-related arrhythmias [1].

Undoubtedly, in the presence of several studies which have demonstrated that OSA increased the all-cause mortality $[4,10,11]$, the treatment of OSA is a significant consideration in any patient with sleep-disordered breathing. Moreover, data from the literature also support the association of OSA with the incidence and morbidity of coronary heart disease, heart failure, arrhythmia, hypertension, and even stroke [9]. In a small single-center prospective study, approximately half the patients with OSA (11 patients; 47\%) presented severe cardiac rhythm disturbances, which were significantly reduced by CPAP [3]. Interestingly, a recent large, randomized trial showed different results. In this study (SAVE study) [13], CPAP plus usual care, as compared with usual care alone, did not prevent cardiovascular events in patients with moderateto-severe OSA and established cardiovascular disease. However, CPAP significantly improved health-related quality of life and mood, reducing the daytime sleepiness and snoring. Last but not least, there is no study available in the literature to evaluate the use of CPAP or other treatment options for OSA in reducing SCD.

In their review article, Roth et al. [7] searched the literature for data reporting the impact of OSA on cardiac arrhythmias, and they aim to demonstrate the patho- physiological mechanisms associated with OSA and SCD (intermittent nocturnal hypoxia, autonomic function, ion channel remodeling, electrolyte shifts, and others). The authors conclude that OSA should be included in the risk assessment for SCD by the Russian National Society of Arrhythmology as an independent risk factor. Although there is no strong evidence to support this conclusion, the data presented by this review article support the fact that OSA is associated with arrhythmogenesis and SCD. The question "if the OSA without cardiovascular disease can cause SCD” remains unanswered though. Hence, more studies are required to arrive at a definite conclusion. OSA should be included in panels of investigations for risk stratification of SCD [1]. The efficacy of newer treatment approaches for OSA such as upper-airway stimulation has already given encouraging results [14], and novel techniques are under investigation. Till then, OSA should be considered as a potential independent risk factor for SCD in patients with or without established cardiovascular disease and should be evaluated in any patient with suspicion of sleep-discorded breathing.

\section{Disclosure Statement}

The authors declare no conflicts of interest.

References

1 Priori SG, Blomstrom-Lundqvist C, Mazzanti A, Blom N, Borggrefe M, Camm J, et al: 2015 ESC Guidelines for the management of patients with ventricular arrhythmias and the prevention of sudden cardiac death: The task force for the management of patients with ventricular arrhythmias and the prevention of sudden cardiac death of the European Society of Cardiology (ESC). Endorsed by: Association for European Paediatric and Congenital Cardiology (AEPC). Eur Heart J 2015; 36:2793-2867.

2 Zhang L, Hou Y, Po SS: Obstructive sleep apnoea and atrial fibrillation. Arrhythm Electrophysiol Rev 2015;4:14-18.

3 Simantirakis EN, Schiza SI, Marketou ME, Chrysostomakis SI, Chlouverakis GI, Klapsinos NC, et al: Severe bradyarrhythmias in patients with sleep apnoea: the effect of continuous positive airway pressure treatment: a long-term evaluation using an insertable loop recorder. Eur Heart J 2004;25:1070-1076.

4 Gami AS, Olson EJ, Shen WK, Wright RS, Ballman KV, Hodge DO, et al: Obstructive sleep apnea and the risk of sudden cardiac death: a longitudinal study of 10,701 adults. J Am Coll Cardiol 2013;62:610-616. 
5 Gami AS, Howard DE, Olson EJ, Somers VK: Day-night pattern of sudden death in obstructive sleep apnea. N Engl J Med 2005;352: 1206-1214

6 Zeidan-Shwiri T, Aronson D, Atalla K, Blich M, Suleiman M, Marai I, et al: Circadian pattern of life-threatening ventricular arrhythmia in patients with sleep-disordered breathing and implantable cardioverter-defibrillators. Heart Rhythm 2011;8:657-662.

7 Brodovskaya T, Grishina I, Peretolchina T, Solenskaia O, Kovtun O, Teplyakova O, Chernjadev S, Popov A, Kurmin V: Clues to the pathophysiology of sudden cardiac death in obstructive sleep apnea. Cardiology 2018, DOI: $10.1159 / 000490308$.
8 Franklin KA, Lindberg E: Obstructive sleep apnea is a common disorder in the population - a review on the epidemiology of sleep apnea. J Thorac Dis 2015;7:1311-1322.

9 Javaheri S, Barbe F, Campos-Rodriguez F, Dempsey JA, Khayat R, Javaheri S, et al: Sleep apnea: types, mechanisms, and clinical cardiovascular consequences. J Am Coll Cardiol 2017;69:841-858.

10 Marshall NS, Wong KK, Liu PY, Cullen SR, Knuiman MW, Grunstein RR: Sleep apnea as an independent risk factor for all-cause mortality: the Busselton Health Study. Sleep 2008; 31:1079-1085.

11 Young T, Finn L, Peppard PE, Szklo-Coxe M, Austin D, Nieto FJ, et al: Sleep disordered breathing and mortality: eighteen-year follow-up of the Wisconsin sleep cohort. Sleep 2008;31:1071-1078.

12 Young T, Palta M, Dempsey J, Skatrud J, Weber S, Badr S: The occurrence of sleep-disordered breathing among middle-aged adults. N Engl J Med 1993;328:1230-1235.

13 McEvoy RD, Antic NA, Heeley E, Luo Y, Ou Q, Zhang X, et al: CPAP for prevention of cardiovascular events in obstructive sleep apnea. N Engl J Med 2016;375:919931.

14 Strollo PJ Jr, Soose RJ, Maurer JT, de Vries N, Cornelius J, Froymovich O, et al: Upper-airway stimulation for obstructive sleep apnea. N Engl J Med 2014;370:139-149. 